\title{
Efficient Light Confinement with Nanostructured Optical Microfiber Tips
}

\author{
Ming Ding, ${ }^{* 1}$ Oliver Fenwick, ${ }^{2}$ Francesco Di Stasio, ${ }^{2}$ Jun-Yu Ou, ${ }^{1}$ Neil Sessions, ${ }^{1}$ Yongmin \\ Jung, ${ }^{1}$ Franco Cacialli, ${ }^{2}$ Gilberto Brambilla ${ }^{1}$ \\ ${ }^{I}$ Optoelectronics Research Centre, University of Southampton, Southampton SO17 1BJ, United Kingdom \\ ${ }^{2}$ Department of Physics and Astronomy and London Centre for Nanotechnology, University College London, London \\ WCIE 6BT, United Kingdom \\ *md20g09@orc.soton.ac.uk \\ Tel: +44(0)2380593143 Fax: +44(0)2380593142
}

\begin{abstract}
Nanostructured optical microfiber tips are proposed and experimentally demonstrated to efficiently confine light beyond the diffraction limit at high powers. Focused ion beam milling was used for the nanostructuring of gold-coated optical microfiber tips with both single-ramp and wedge geometries. Small apertures were formed by flat cutting or hole drilling and optical spot sizes of $\sim \lambda 10$ with high transmission efficiency were achieved. Numerical simulations were carried out to optimize the device design with circularly polarized light. Enhanced transmission efficiencies (higher than $10^{-2}$ ) were recorded by optimizing the overall light throughput along the fiber tips. The tip thermal behavior was investigated by launching high powers into the device and recording the tip position in a scanning optical near-field microscopy set-up. This nanostructured optical microfiber tip has the potential for applications in optical recording, scanning near-field optical microscopy and lithography.

Keywords: light confinement, focused ion beam, spot size, transmission efficiency
\end{abstract}

\section{Introduction}

Since Tong and coworkers published their seminal paper in Nature in 2003 [1], interest in optical microfibers has increased continuously, finding a multitude of applications [2] in a variety of devices ranging from high-Q resonators to sensors [3]. Microfibers exhibit many interesting attributes, which include configurability, flexibility, large evanescent fields, bio compatibility and strong light confinement. Among these, the most attractive is possibly the capability to confine light to the diffraction limit for lengths that are thousands of times longer than the Rayleigh length. While in free space Gaussian beams the Rayleigh criterion limits the maximum 
confinement length to few microns, in optical microfibers confinement lengths can be well in excess of meters and are limited only by the microfiber propagation loss.

The diffraction limit is the generally accepted limitation to optical imaging and focusing systems: the minimum achievable spot size $\left(\omega_{0}\right)$ is related to the wavelength $(\lambda)$ of the propagating light and to the refractive index $(n)$ of the medium where light is focused by [4]:

$$
\omega_{0} \geq \frac{\lambda}{2 n}
$$

To overcome the diffraction limit, several techniques have been investigated, including metamaterials [5-9], high index contrast waveguides [10], photonic crystal fibers [11], micro/nanofiber array [12], tapered microtube [13], longitudinal evanescent waves/scanning near-field optical microscopy (SNOM) [14-16], and surface plasmons (SPs) [17-20].

Negative refractive index materials were firstly proposed by Veselago in 1968 [21] as means capable to focus electromagnetic radiation well below the diffraction limit. In the late 1990s, Pendry [22] proposed that electromagnetic resonances in artificially engineered metamaterials made of metal loops and wires could reach values not accessible in natural materials; this was experimentally realized in 2000 at microwave frequencies $(\sim 10 \mathrm{GHz})$ [23, 24]. Although extraordinary resolution has been achieved with these metamaterials, no light localization within a small spot size has been demonstrated.

High index contrast waveguide have been proposed by $\mathrm{Xu}$ and collaborators in 2004 [10] in the form of a "slot-waveguide" structure where a low-index, nanometer-wide area was embedded in a high index material medium: because of the discontinuity of the electric field at the high index contrast interface the optical field was enhanced and confined in the low refractive index material. Later, Wiederhecker [11] demonstrated field concentration within a sub-wavelength scale air hole running down the length of photonic crystal fibers core. The core resembles a submicrometer diameter tube with a bore diameter of $200 \mathrm{~nm}$ or less. In such a small waveguide, guided light is not tightly confined within the high index glass, but also extends into the low index material surrounding it. The evanescent light decaying into the central hole decays only a little before meeting the opposite side, as long as the hole is sufficiently small. This ensures that 
the electric field inside the bore is stronger than outside it, in the silica. Yet, this technique allowed only for a small fraction of the power to be confined in the low refractive index material, and, above all, confinement efficiency is low when the spot size becomes smaller than $100 \mathrm{~nm}$.

A structure based on-micro/nanofiber arrays has been used to focus optical beams with subwavelength resolution in the far field [12]. By optimizing the structure with numerical simulations, spots with full-width-at-half-maximum (FWHM) of $0.43 \lambda$ at a distance of $9 \lambda$ from the output end face of the micro/nanofiber array was obtained. Sub-wavelength far field focusing was also proposed with a tapered microtube fabricated from a glass capillary [13]. Experimental results revealed a smallest spot, with a near diffraction-limited FWHM of $0.65 \lambda$, was positioned at a distance of $2.2 \lambda$ from the output end face of the microtube. Although these two techniques allow for far field focusing, they provide only limited confinement, which is typically few hundreds nanometers.

Longitudinal evanescent fields have been exploited to obtain sub-wavelength confinement of light in metal-coated tips and found applications in SNOM systems [25-29]. Yet, SNOM probes have very low transmission efficiencies, e.g. $10^{-3} \sim 10^{-6}[26,30]$, as they require small apertures and small tapering angles, which results in a strong decay in the evanescent field intensity. Also, they usually can only stand relatively small powers, typically of the order of $1 \mathrm{~mW}$.

Devices based on surface plasmons have been proposed and experimentally demonstrated for efficient sub-wavelength light confinement, but they always dealt with small powers and mostly in planar geometries. In 2007, Ding [31] proposed an apertureless silver-coated optical fiber tip in which the radially polarized waveguide modes of an optical fiber taper was converted into the plasmons propagating at the outer surface of the tip. Although they are predicted to have extremely high transmission efficiency, apertureless optical fiber tips with high transmission efficiency have never been manufactured $[32,33]$. On the contrary, apertured tips have been widely used in light nano-focusing, albeit without the aid of the surface plasmons [26, 34-36]. In 2009, apertured tips exploiting the surface plasmons to confine light to sub-wavelength spot sizes $(\lambda / 3)$ in optical fibers and microfibers were realized $[37,38]$ : the use of the SPs improved transmission efficiency by several orders of magnitude. Yet, the use of SPs limits the minimal 
spot size, as the radial component of the evanescent field becomes increasingly more important for reducing aperture sizes.

In this paper, an efficient sub-wavelength light confinement source capable to focus relatively high powers is proposed by nanostructuring optical microfiber tip. Its design for the case of circularly polarized light is discussed and some examples of fabrication shown.

High transmission efficiency and high power are fundamental requirements for many applications, which include amongst others data recording, imaging and photolithography.

\subsection{Data recording}

Data storage on disks is being achieved mainly in two ways, using either optical or magnetic recording.

Optical storage relies on the phase change of a glassy (mainly chalcogenide) thin layer of a disc exposed to a focused laser beam. The CD-ROM (Compact Disc - Read Only Memory) was the first important example of optical storage and it used a $1.2 \mathrm{~mm}$ thick disc of polycarbonate plastic with a thin layer of aluminum to store up to $703.1 \mathrm{MB}$ of data using a $780 \mathrm{~nm}$ laser. Few years later, the DVD-ROM (Digital Versatile Disc-Read Only Memory) used a shorter wavelength $(\lambda \sim 650 \mathrm{~nm})$ to phase change a smaller area of the media surface compared to CDs $(1.32 \mu \mathrm{m}$ versus $1.6 \mu \mathrm{m}$ for $\mathrm{CD}$ ), allowing for a storage capability of $4.7 \mathrm{~GB}$ for a single-sided single layer and 8.54 GB for a single-sided double layer. 2006 saw the release of the Blu-ray Disc (BD) which can store up to $100 \mathrm{~GB}$. The improvement in the data storage was made possible mainly by the use of a semiconductor laser diode with a reduced wavelength of $405 \mathrm{~nm}$ and a high refractive index lens. All existing methods to improve the storage capability in $\mathrm{CD}$, DVD and BD rely on the shift of the diffraction limit to smaller sizes by reducing the laser wavelength and increasing the refractive index. Multilayer [39, 40] and holographic [41, 42] recording are being investigated as an alternative route. Sub-wavelength light confinement can be used to improve further the storage capability. If spot sizes smaller than $20 \mathrm{~nm}$ are used, storage in excess of $1 \mathrm{~TB}$ can be predicted on a single disk. 
Small spot-size sources can also benefit magnetic recording. In all-optical magnetic recording (AOMR), to obtain the high storage capability optical energy must be delivered efficiently to reverse the magnetization in a spot on the medium that is much smaller than the diffraction limit. In AOMR, circular polarized light should have the ability to act upon a magnetic system in a way similar to a magnetic field directed parallel to the wave vector of the light via the inverse Faraday effect $[43,44]$. Moreover, right- and left-handed circularly polarized waves should act as magnetic fields of opposite sign. This effect was only recently confirmed experimentally $[45,46]$ and has also been demonstrated to be an efficient tool for coherently controlling the small angle processional magnetization dynamics in magnetic dielectrics [47, 48].

\subsection{Near-field optical imaging}

Near-field optical imaging, usually referred as SNOM, is another important application of fiberized sub-wavelength sources [49]. This technique is used for nanostructure investigation which breaks the observed far-field resolution limit by exploiting the properties of evanescent waves of the near-field. This is done by placing the probe very close to the specimen surface (typically in the region of $10 \mathrm{~nm}$ ) and allows for surface inspection with high spatial, spectral and temporal resolution. In this technique, the image resolution is limited by the size of the probe aperture and not by the wavelength of the illuminating light. Light from a laser is sent to a fiber and then to a sub-wavelength aperture, in the tapered end, which is positioned very near to the sample [15]. Collecting the reflected or transmitted light with a normal optical system and with a light detector, it is possible to achieve image resolutions better than $100 \mathrm{~nm}$ [26]. The scanning tip, depending upon the operation mode, is usually a tapered or etched optical fiber coated with metal $[26,50,51]$ or a standard Atomic Force Microscope (AFM) cantilever with a hole in the center of the pyramidal tip [52].

\subsection{Photolithography}

Photolithography is a process used in microfabrication to selectively remove parts of a thin film (or of a substrate). It uses light to transfer a geometric pattern from a photomask to a light sensitive chemical (photoresist) on the substrate. In order to have more complex integrated 
circuits $[53,54]$, the aim of photolithography is to reduce the feature sizes as much as possible. In previous years, the technological limit has been related to the focusing of light and the way forward was to reduce the wavelength of the light used for the exposure of the photoresist. As a result, this method is subject to the diffraction limit. Current state-of-the-art photolithography tools use deep ultraviolet light with wavelength $193 \mathrm{~nm}$ and immersion lithography and/or mask shifting, which allow minimum feature sizes down to $32 \mathrm{~nm}$ [55]. A fiberized sub-wavelength light source can be used to overcome diffraction limit and it can be used in the photolithography world in three ways.

One consists in directly "writing" the photoresist layer [56,57]. This technique of direct writing and a fiberized sub-wavelength light confinement source can be conceived like a "pen". Using a matrix of pens the exposure time for the wafer can be substantially reduced. The second application of sub-wavelength light sources is for mask repair. Masks that are used for photolithography are extremely expensive. The possibility to have a light source to repair a mask with a sufficient detail can be increase the mask life span, thus decrease its overall cost. Finally, another important application is the diagnosis of defects. In fact, at the moment, there are no ways to investigate defects with size smaller than $22 \mathrm{~nm}[58,59]$.

In some cases, for example for organic semiconductors (OS), it is also possible to write directly the final semiconductors nanostructures by exposure to optical evanescent ways without the use of a sacrificial resist [36].

\section{Device design}

In this manuscript, nanostructured microfiber tips are optimized to improve transmission efficiency at high transmitted powers.

Three structures have been investigated: the single-ramp (Fig. 1 (a)), where one surface was cut at a specific angle $\alpha$; the wedge (Fig. 1 (b)), where two surfaces were cut with the same slope within the instrument resolution; and the cone (Fig. 1 (c)), which can exploit the various polarization directions when circularly polarized light is used. While the first design is easier to fabricate, the second and third designs offer a shorter light path for a given microfiber diameter: 
since in coated sub-wavelength diameter waveguides light propagation is not permitted, only a longitudinal evanescent field exists. The evanescent wave experiences exponential attenuation along its path, thus a shorter path assures higher transmission efficiency. $\alpha$ was chosen to optimize transmission and beam confinement.

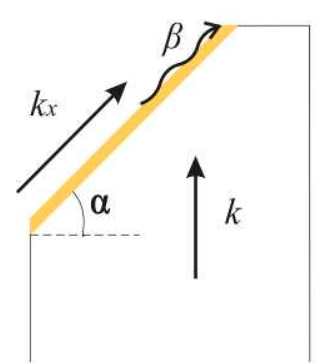

(a)

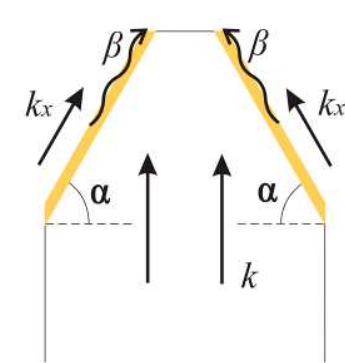

(b)

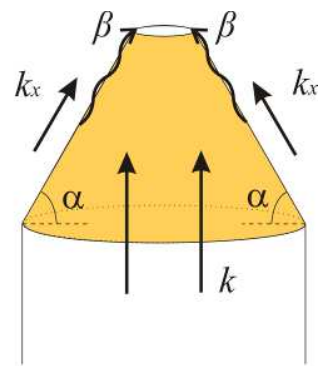

(c)

Fig. 1 Schematic of (a) single-ramp sample, (b) wedge sample and (c) cone sample. $\alpha$ represents the slope angle. $\beta, k$ and $k_{x}$ represent the surface plasmon propagation constant, the light wave vector and its component along $\beta$, respectively.

Simple mathematical manipulations show that SPs can be excited when transversely magnetic polarized beams hit the interface between the metal (gold in this paper) and dielectric at a specific resonance angle $\alpha$. Resonance occurs when the projection of the light wave vector on the newly formed surface $\left(k_{x}=k \cdot \sin \alpha\right)$ equals the SPs propagation constant $\beta$ in the same direction (Fig. 1). The resonance condition can be expressed as:

$$
\alpha=\arcsin \left(\frac{1}{n_{\text {eff }}} \sqrt{\frac{\varepsilon_{\text {gold }} \varepsilon_{\text {air }}}{\varepsilon_{\text {gold }}+\varepsilon_{\text {air }}}}\right)
$$

where $n_{\text {eff }}$ is the effective index of the mode which decreases for reducing microfiber diameter, $\varepsilon_{\text {gold }}$ and $\varepsilon_{\text {air }}$ are the dielectric constants of gold and air, respectively. Although SP can aid the power confinement, they also exhibit a radial exponential dependence, which limits the ultimate tight confinement over small spot sizes. For this reason designing the slope angle $\alpha$ is the most important feature for efficient tight confinement. Fig. 2 shows the dependence of $\alpha$ on the microfiber tip diameter and on the wavelength for an optimal SP excitation. Fig. 2 (a) shows that $\alpha$ varies from $44^{\circ}$ to $90^{\circ}$ for tip diameters $D$ ranging from $0.3 \mu \mathrm{m}$ to $5 \mu \mathrm{m}$. Fig. 2 (b) reveals that $\alpha$ varies more than $10^{\circ}$ in the wavelength range $600-1600 \mathrm{~nm}$ and that the smaller $D$ is, the stronger the dependence of $\alpha$ on the wavelength. As a result, in tips not only $\lambda$ but also $D$ can be used to 
fulfill the resonance condition. In our experiments, microfiber tips with $D \sim 1 \mu \mathrm{m}$ were chosen because they provide a tight confinement, a reasonable stiffness and better tolerance to nanostructuring imprecision: in tips with small diameters a small imprecision (smaller than $0.1^{\circ}$ in the focused ion beam (FIB) system) in $\alpha$ results in a shift of the SP resonance peak wavelength smaller than $100 \mathrm{~nm}$.
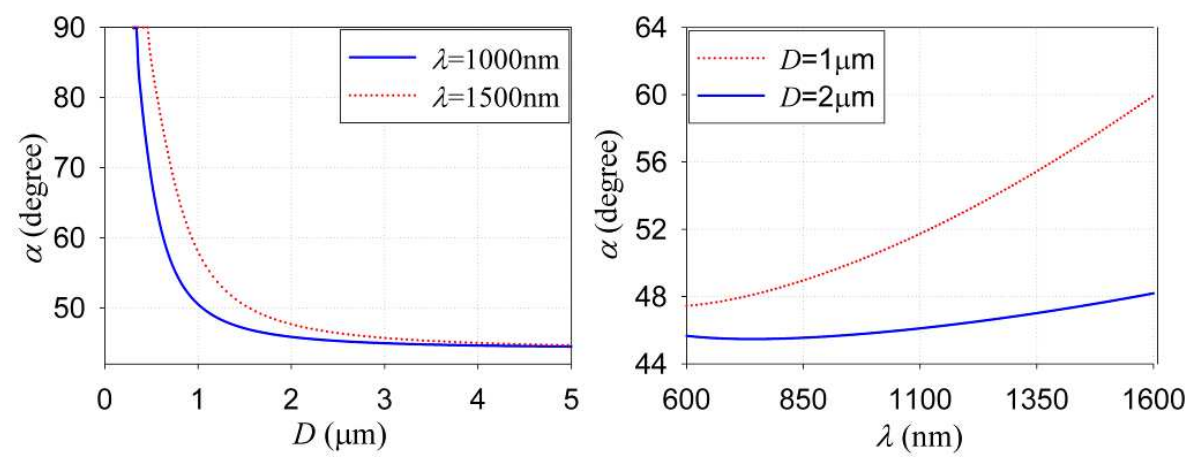

Fig. 2 Dependence of slope angle $\alpha$ on (a) microfiber tip diameter $D$ and (b) wavelength $\lambda$ for an optimal SP excitation.

In order to optimize the microfiber tips design and obtain high transmission efficiencies, theoretical analysis were carried out using 3D Finite Element Method (FEM) to numerically solve Maxwell equations in the frequency domain. The numerical model was defined, solved and analyzed using the commercial software COMSOL 4.1 Multiphysics. Since in AOMR the circularly polarized light is the most favorable polarization - it leads to three desirable features for the optically-induced magnetization: a relatively high spatial confinement, a direction nearly perpendicular to the opto-magnetic medium and a large magnitude $[60,61]-$, a single mode with circular polarization is launched into the microfiber tip conical cut structure shown in Fig. 3. The microfiber, made from a telecom single mode fiber (SMF-1300/1550-9/125-0.25-L (OZ optics, Canada) which has $\sim 8.2 \mu \mathrm{m}$ core diameter, $\sim 125 \mu \mathrm{m}$ cladding diameter, $\sim 0.12$ numerical aperture and $1250 \mathrm{~nm}$ cut-off wavelength), has $1 \mu \mathrm{m}$ diameter and was operated at the input wavelength $\lambda=800 \mathrm{~nm}$.; a layer of gold is coated on the microfiber tip and an aperture is opened at its apex. The tip is surrounded by air in a box aimed to avoid reflections from the boundary. The chosen boundary conditions were: scattering boundary condition in the exterior boundary and continuity boundary condition in the interior boundary. Simulations were run with controlled mesh size (50 
$\mathrm{nm}$ in silica, $10 \mathrm{~nm}$ in gold coating and $100 \mathrm{~nm}$ in air surrounding) to make efficient use of computer memory.

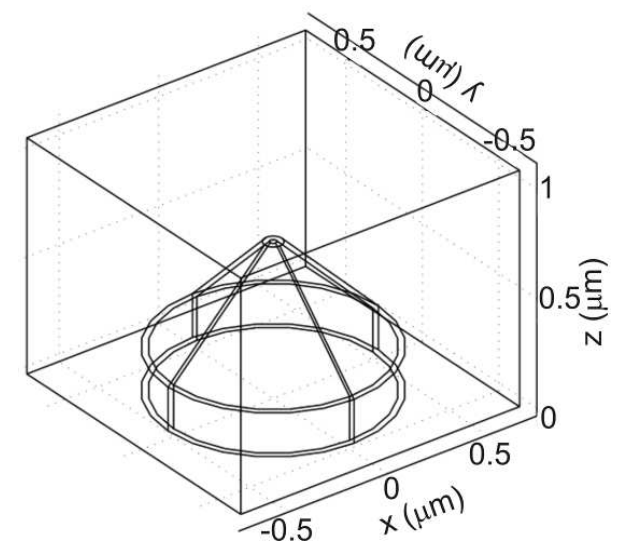

Fig. 3 Geometry of the modelled conical cut structure.

Firstly, the modeling assumed a coating thickness $t=20 \mathrm{~nm}$, an aperture diameter $d=30 \mathrm{~nm}$, and a slope angle of $\alpha=48.713^{\circ}$. This angle was chosen to take advantage of SPs for energy confinement. The refractive index of gold, silica and air were assumed to be $0.1808-5.11731 \mathrm{i}$, 1.444, and 1.00054 at the wavelength $\lambda=800 \mathrm{~nm}$, respectively. Fig. 4 shows the electric field distribution across the $\mathrm{x}-\mathrm{z}$ plane passing through the center of the aperture. From the electric field plot, the field experiences a considerable enhancement at the tip apex. It is also evident that strong radial evanescent fields are present outside the tip for $\mathrm{z} \sim 0.6 \mu \mathrm{m}$.

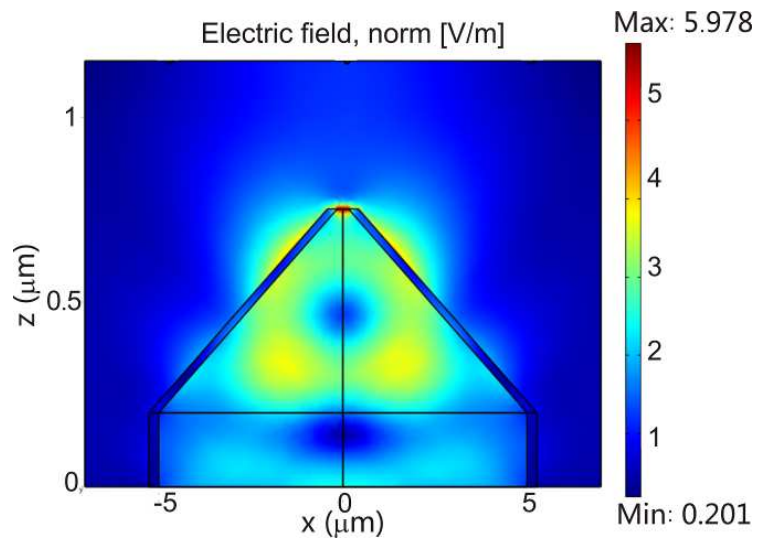

Fig. 4 Electric field distribution across the $\mathrm{x}-\mathrm{z}$ plane passing through the structure center. Coating thickness, aperture diameter and slope angle were $t=20 \mathrm{~nm}, d=30 \mathrm{~nm}$ and $\alpha=48.713^{\circ}$, respectively.

In optical recording, as in SNOM and photolithographic applications, the working distance is of the order of few nanometers. The 2D electric near-field on the $x-y$ plane of $5 \mathrm{~nm}$ above the 
apex is showed in Fig. 5 (a). The field is observed to be highly localized. The 3D image of the electric near-field distribution is also plot using MATLAB (Fig. 5 (b)). Finally, a cross section of the electric field intensity at $\mathrm{y}=0$ is presented in Fig. 5 (c); the FWHM which can be used to characterize the device spot size was calculated from this plot to be $44.7 \mathrm{~nm}$, i.e. $\lambda / 17$. Although the SP radial evanescent field could represent a limitation to field concentration, Fig. 5 (c) shows that in this case its field strength is well below $30 \%$ of the maximum.

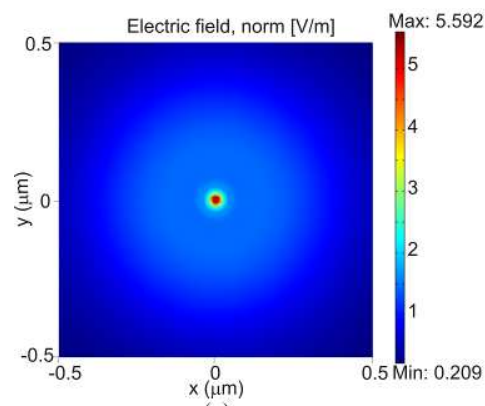

(a)

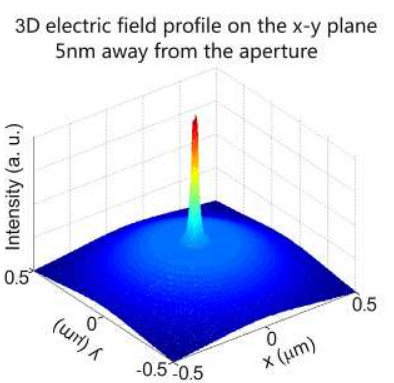

(b)

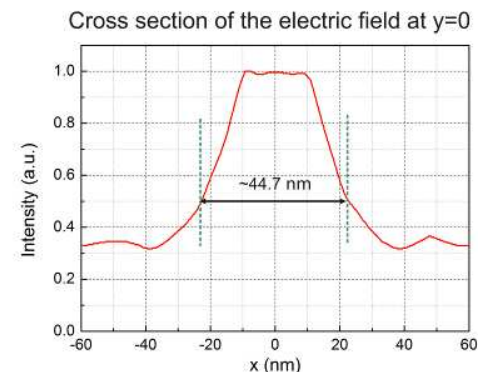

(c)

Fig. 5 (a) 2D electric near-field distribution at $x-y$ plane of $5 \mathrm{~nm}$ above the aperture for a tip with slope $\alpha=48.713^{\circ}$ and $30 \mathrm{~nm}$ aperture. (b) 3D electric near-field distribution at the $\mathrm{x}-\mathrm{y}$ plane of $5 \mathrm{~nm}$ above the aperture. (c) A cross section of the electric field intensity at $\mathrm{y}=0$.

In order to identify whether a smaller spot size can be achieved with the designed structure, the modeling for a $5 \mathrm{~nm}$ aperture size was carried out. Fig. 6 (a) and (b) show that in this case the electric field is localized due to the aperture but a strong evanescent field can be observed for distances larger than $20 \mathrm{~nm}$. Fig. 6 (c) (cross section of the electric field at $y=0$ ) shows that the evanescent field outside the aperture is above 50\%, thus will affect the efficient confinement.

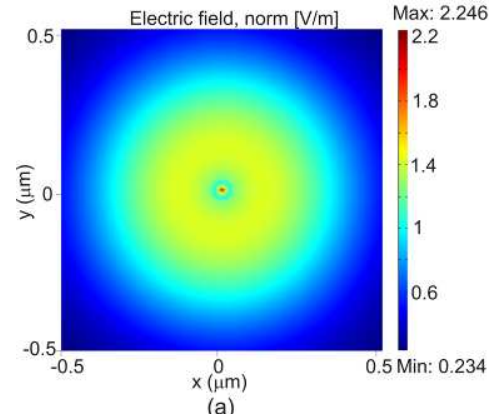

$x(\mu \mathrm{m})$
$(\mathrm{a})$

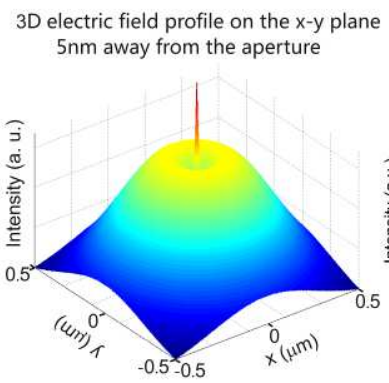

(b)

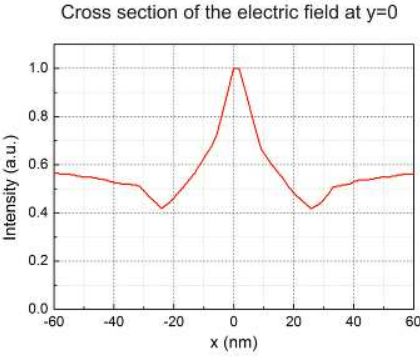

(c)

Fig. 6 (a) 2D electric near-field distribution on an $x-y$ plane $5 \mathrm{~nm}$ far from the aperture for microfiber tip with $\alpha=48.713^{\circ}$ and a $d=5 \mathrm{~nm}$ aperture. (b) $3 \mathrm{D}$ electric near-field distribution on the $\mathrm{x}-\mathrm{y}$ plane, $5 \mathrm{~nm}$ from the microfiber tip aperture. (c) A cross section of the electric field intensity at $\mathrm{y}=0$. 
The tip's transmission efficiency in simulations was defined as the absolute value of the ratio between the Poynting vector integral at the input and output planes (only the section above the aperture), and resulted to be several orders of magnitude larger than what was observed in common SNOM tips [26].

Fig. 7 shows the transmission efficiency variation dependence on the slope angle for $30 \mathrm{~nm}$ and $5 \mathrm{~nm}$ aperture size, revealing that smaller slopes are associated with higher transmission efficiencies. High transmission efficiency is the results of two main factors. When the microfiber tip diameter becomes smaller than a cut-off value (typically $\lambda / 3$ in silica), no modal propagation occurs and only evanescent modes with a field decaying exponentially along the longitudinal direction (towards the aperture) exist. The longer is the distance between the position of the cutoff diameter and the aperture, the smaller is the transmission efficiency. On the other hand, when plasmons are excited at the interface with gold transmission efficiency can be improved. Fig. 7 shows that this latter contribution is negligible, as SP excitation is strongly angle dependent and no evident peaks have been observed at specific angles.

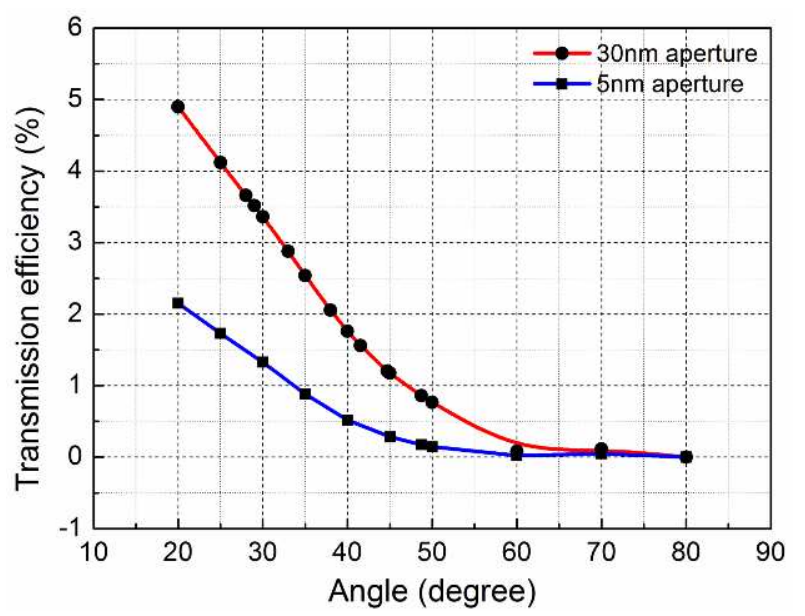

Fig. 7 Transmission efficiency versus slope angle at $\lambda=800 \mathrm{~nm}$. Tip diameter is $D=1 \mu \mathrm{m}$, aperture diameter is $d=30 \mathrm{~nm}$, coating thickness $t=20 \mathrm{~nm}$.

Electric field distributions across the $\mathrm{x}-\mathrm{z}$ plane passing through the structure center for slopes $\alpha=20^{\circ}, 25^{\circ}, 30^{\circ}, 35^{\circ}, 40^{\circ}, 45^{\circ}, 50^{\circ}, 60^{\circ}, 70^{\circ}$, and $80^{\circ}$ are shown in Fig. 8 for aperture size $d=30$ nm (Fig. 8 (a)) and $5 \mathrm{~nm}$ (Fig. 8 (b)). At $\alpha=20^{\circ}$ and 30 , a negligible SP excitation was observed. As the slope angle increases, an increased field localization at the gold /dielectric interface is 
observed, with a maximum being reached at $\alpha=70^{\circ}$. Fig. 7 shows that transmission efficiency decreases for increasing angles and at $\alpha=70^{\circ}$ has an extremely small enhancement compared with $\alpha=60^{\circ}$ and $\alpha=80^{\circ}$. Therefore, although SPs can be excited in the gold-coated microfiber tip, their contribution to transmission efficiency can be considered negligible. Transmission efficiency is mainly dominated by the distance between the position of the cut-off diameter and the microfiber tip aperture.

(a) $d=30 \mathrm{~nm}$
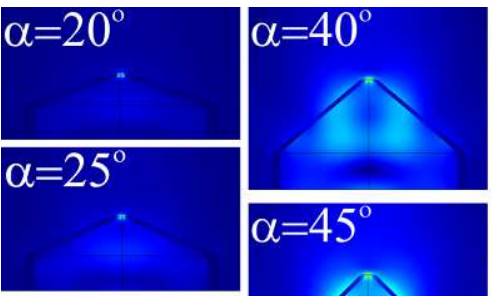

$\alpha=30^{\circ}$
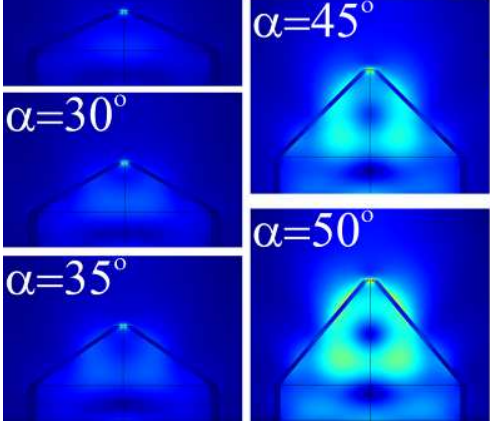

\section{$\alpha=50^{\circ}$}

$\alpha=35$
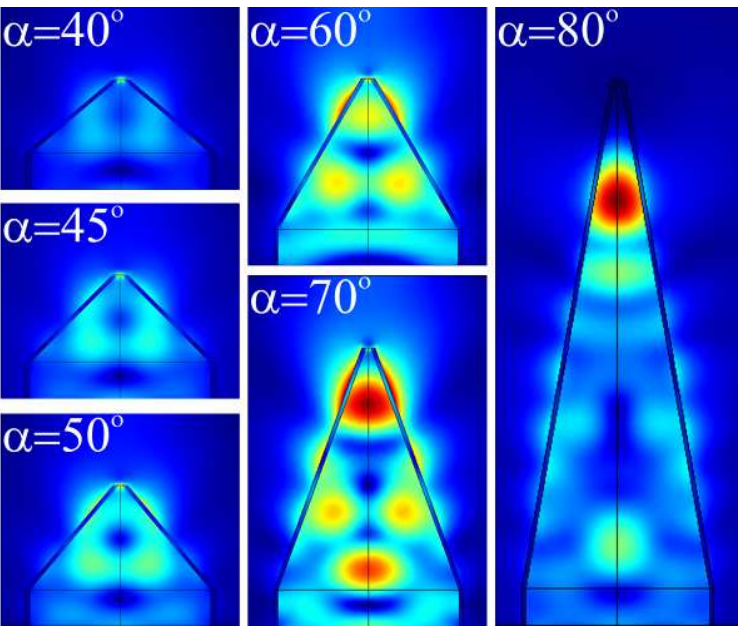

(b) $d=5 \mathrm{~nm}$
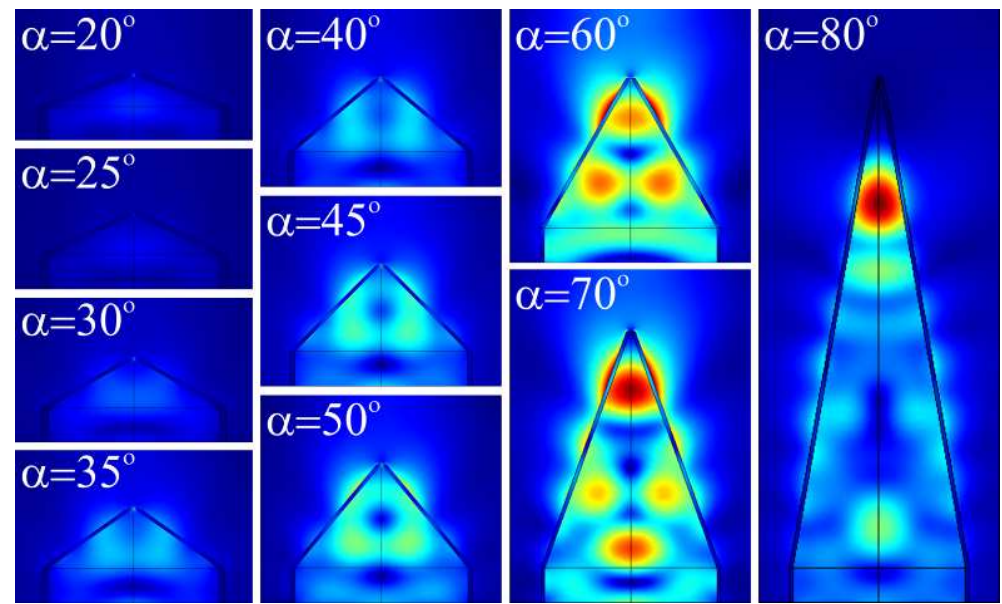

Fig. 8 Electric field distribution across the x-z plane passing through the structure center for slope angle $\alpha=20^{\circ}$, $25^{\circ}, 30^{\circ}, 35^{\circ}, 40^{\circ}, 45^{\circ}, 50^{\circ}, 60^{\circ}, 70^{\circ}$, and $80^{\circ}$. Coating thickness and aperture diameters were $t=20 \mathrm{~nm}$ and (a) $d=30 \mathrm{~nm}$, (b) $d=5 \mathrm{~nm}$ respectively.

Fig. 8 clearly shows that at small angles the contribution of radial evanescent field is small, thus allowing for a better confinement. Fig. 9 shows the simulations carried out assuming a coating thickness $t=20 \mathrm{~nm}$, an aperture diameter $d=5 \mathrm{~nm}$, and a slope angle of $\alpha=20^{\circ}$. In these 
conditions light is confined to $25 \mathrm{~nm}$ spot sizes with a relatively small intensity in the evanescent field. A FWHM of $25.3 \mathrm{~nm}$ was observed (Fig. 9 (c)) which can be decreased by further optimization.

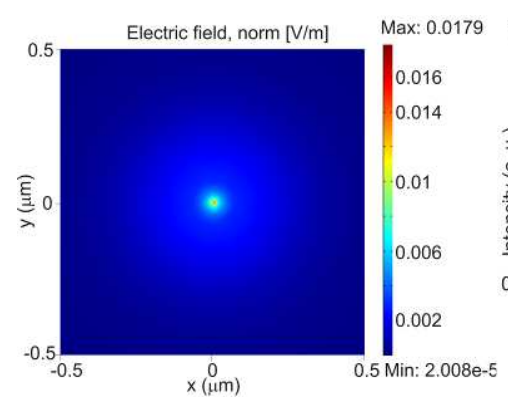

(a)

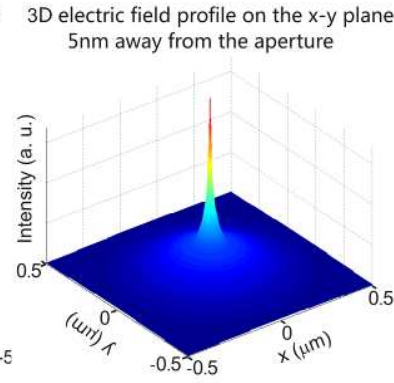

(b)

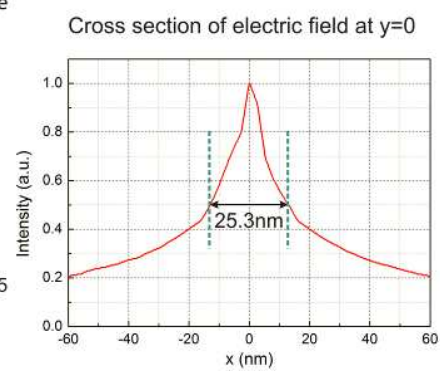

(c)

Fig. 9 (a) 2D electric near-field distribution at $\mathrm{x}$-y plane of $5 \mathrm{~nm}$ above the aperture for $20^{\circ}$ slope angle and 5 $\mathrm{nm}$ aperture size. (b) 3D electric near-field distribution at the $\mathrm{x}-\mathrm{y}$ plane of $5 \mathrm{~nm}$ above the aperture. (c) A cross section of the electric field intensity at $\mathrm{y}=0$.

The transmission efficiency dependence on aperture size is analyzed in Fig. 10, which clearly shows a decrease for decreasing aperture sizes. Although the spot size is determined by the aperture size, smaller aperture sizes are also associated with smaller transmission efficiencies. Yet, Fig. 10 shows that efficiencies as high as $0.5 \%$ can be predicted even for apertures as small as $5 \mathrm{~nm}$.

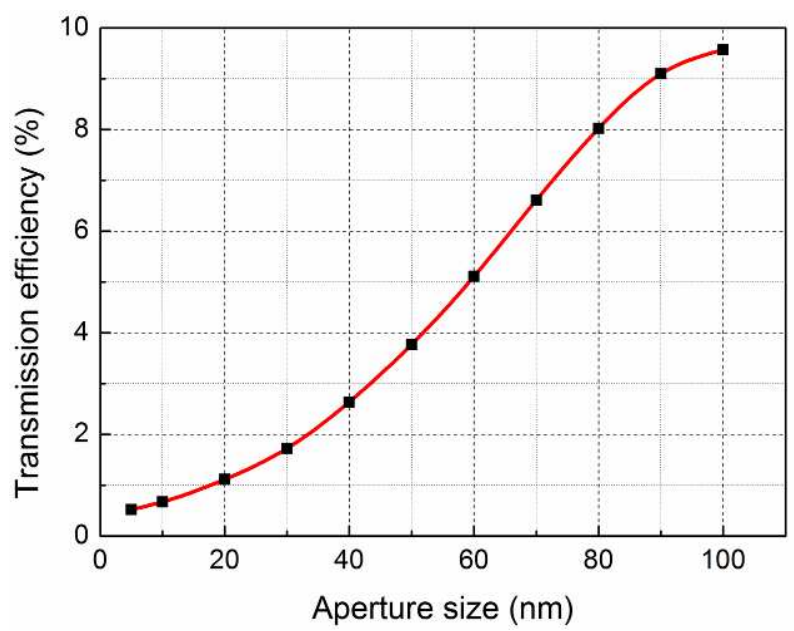

Fig. 10 Transmission efficiency dependence on aperture size. Taper diameter, slope angle, coating thickness and wavelength are $D=1 \mu \mathrm{m}, \alpha=40^{\circ}, t=30 \mathrm{~nm}$ and $\lambda=800 \mathrm{~nm}$, respectively.

Other factors, e.g. metal materials, coating thickness and input light wavelength also play a role in the transmission efficiency and spot size. Fig. 11 shows the simulations carried out to 
compare different metal materials at $\lambda=800 \mathrm{~nm}$. Trends in Fig. 11 are primarily due to the dielectric constants which have greatest influence on the absorption loss. Silver shows the largest enhancement and widest angle tolerance because of its unique dielectric constant. Gold also presents a relatively large enhancement compared with aluminum and platinum. However, silver is easily oxidized in air, whereas gold is much more stable. Thus, gold was chosen as the metal coating material in the experiments. Indeed, high powers tend to increase the tip temperature, facilitating surface oxidation, thus gold appears to be an excellent candidate for its relatively poor tendency to oxidize. At lower wavelength, the transmission efficiency with aluminum would be comparable with others since aluminum has low real part of refractive index.

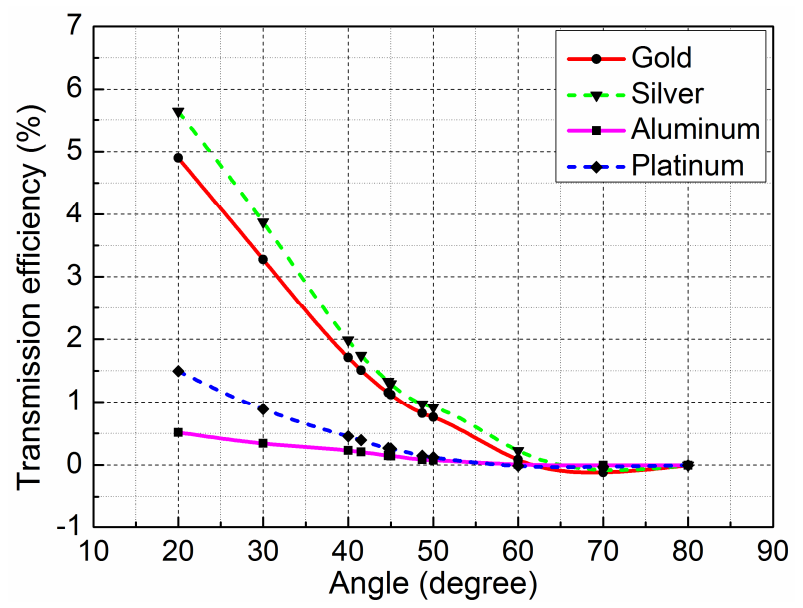

Fig. 11 Transmission efficiency dependence on slope angle for four different coating metals: gold (red solid line), silver (green dash line), aluminium (pink solid line) and platinum (blue dash line). Taper diameter, aperture diameter, coating thickness and wavelength are $D=1 \mu \mathrm{m}, d=30 \mathrm{~nm}, t=20 \mathrm{~nm}$ and $\lambda=800 \mathrm{~nm}$, respectively.

Fig. 12 shows the dependence of transmission efficiency on the coating thickness in the range $15 \mathrm{~nm}$ to $100 \mathrm{~nm}$ at $800 \mathrm{~nm}$ wavelength. The tip diameter, aperture size and slope angle are $D=1 \mu \mathrm{m}, d=30 \mathrm{~nm}$, and $\alpha=40^{\circ}$, respectively. The maximum at $t \sim 30 \mathrm{~nm}$ allows for more fabrication tolerance and a reduction of charging issues during FIB milling. A possible explanation for the transmission efficiency decrease for $t<30 \mathrm{~nm}$ is that at small gold coating thicknesses a considerable fraction of powers lies in the radial evanescent field outside the gold coating and it is not included in the transmission efficiency calculation. This effect is also deleterious for the beam size, which is increased. As the longitudinal evanescent filed overlaps 
with the gold coating, when the coating thickness increases above $30 \mathrm{~nm}$, the overall absorption increases, thus transmission efficiency decreases. Thickness of $t \sim 30 \mathrm{~nm}$ has therefore been used in the experiments.

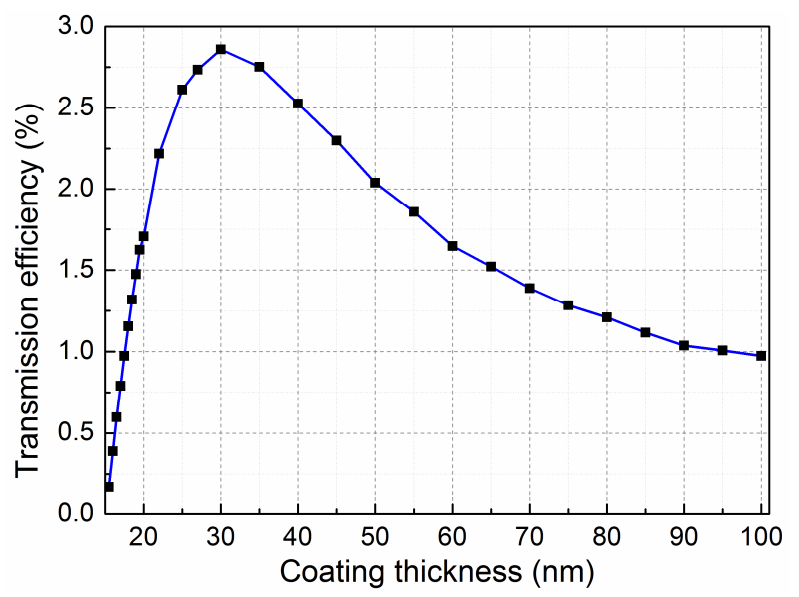

Fig. 12 Microfiber tip transmission efficiency versus gold coating thickness. The taper diameter, aperture diameter, slope angle and wavelength are $\mathrm{D}=1 \mu \mathrm{m}, \mathrm{d}=30 \mathrm{~nm}, \alpha=40^{\circ}$ and $\lambda=800 \mathrm{~nm}$.

Fig. 13 presents the transmission efficiency dependence on the input wavelength when tip diameter, aperture diameter, slope angle and coating thickness are $1 \mu \mathrm{m}, 30 \mathrm{~nm}, 40^{\circ}$ and $30 \mathrm{~nm}$, respectively. The influence of wavelength is rather complicated. Firstly, the dispersion of gold dielectric constant varies considerably with the wavelength [62]. Secondly, the cut-off diameter changes with the wavelength causing a change in the extent of its evanescent field.

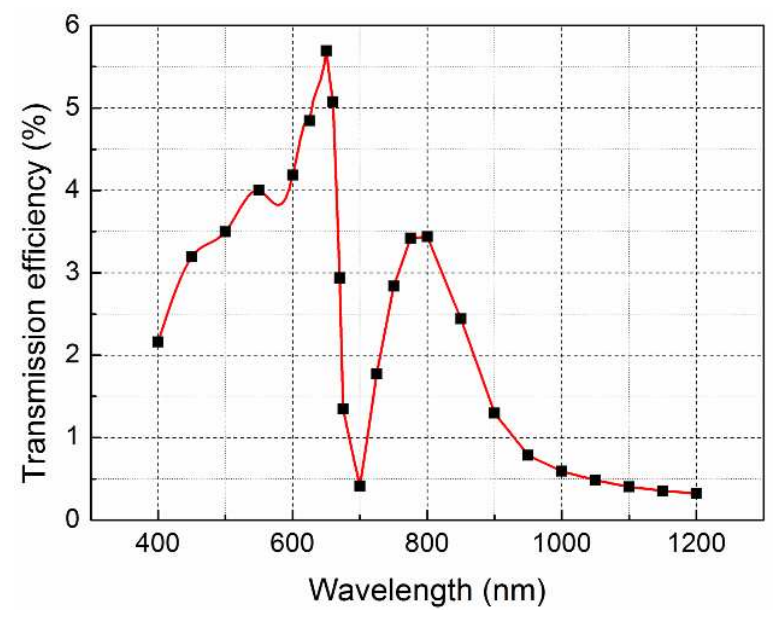

Fig. 13 Transmission efficiency dependence on the wavelength. Taper diameter, aperture diameter, slope angle and coating thickness are $D=1 \mu \mathrm{m}$, is $d=30 \mathrm{~nm}, \alpha=40^{\circ}$ and $t=30 \mathrm{~nm}$, respectively. 
It is known that when the features become smaller than a certain limit the non-locality of the dielectric function should be taken into account [63]. According to ref. [64], for a metal-silicametal waveguide, the plasmon modes undergo blue shift exceeding $10 \%$ at metal thicknesses below 5 angstrom because of the non-locality of dielectric function and there is no obvious shift when the thickness is larger than $\sim 6 \mathrm{~nm}$. Since the gold thickness is chosen $\sim 30 \mathrm{~nm}$ in the design, the non-local effect can be neglected. Moreover, electromagnetic field enhancement between gold nanoparticle dimers is well approximated by the localised model above $\sim 1 \mathrm{~nm}$. Since the apertures considered in the paper are never less than $5 \mathrm{~nm}$, the field enhancement near the aperture is also well approximated by the localised model.

\section{Device fabrication}

Devices have been fabricated using the cleanroom facilities available at the Optoelectronics Research Centre (Southampton, U.K.). The fabrication process can be divided into four main steps: (i) manufacture of optical microfiber tips, (ii) tip milling, (iii) gold layer deposition, and (iv) aperture opening at the coated fiber tip apexes. Fig. 14 shows the schematic diagram of the proposed device fabrication process.

Firstly, microfiber tips were manufactured (Fig. 14 (a)) using a commercial micropipette puller (P-2000) (SUTTER INSTRUMENT Inc., Novato, USA) which is a $\mathrm{CO}_{2}$ laser based pulling machine controlled by a microprocessor. The default configuration of the P-2000 allows the fabrication of microfiber tips with extremely small diameters: typically, fibers can be pulled from $125 \mu \mathrm{m}$ down to $40 \mathrm{~nm}$ diameters and have a taper length of about $1 \mathrm{~mm}$. Since high transmission efficiency is targeted in this paper, the taper angle of the optical fiber tips has to be small enough to adiabatically convert the fundamental mode in the fiber core into a fundamental mode in the microfiber. Love proposed a criterion for adiabaticity [65] based on the coupling length between the fundamental mode and the dominant coupling mode. This condition results in the local tapering angle $\Omega$ being limited by the adiabatic angle $\bar{\Omega}$, defined as a function of the propagation constants of two lowest order modes $\left(k_{1}\right.$ and $\left.k_{2}\right)$ and of the local diameter $(D)$ in the guiding structure: 


$$
\bar{\Omega}=\frac{D\left(k_{1}-k_{2}\right)}{4 \pi}
$$

If $\Omega<\bar{\Omega}$, tapers are adiabatic. Transmissions larger than $98 \%$ can be obtained in adiabatic fiber tapers [66]. $\mathrm{CO}_{2}$ laser power, pulling speed, tension and delays were optimized to maximize the adiabaticity of the transition region between the optical fiber and microfiber.

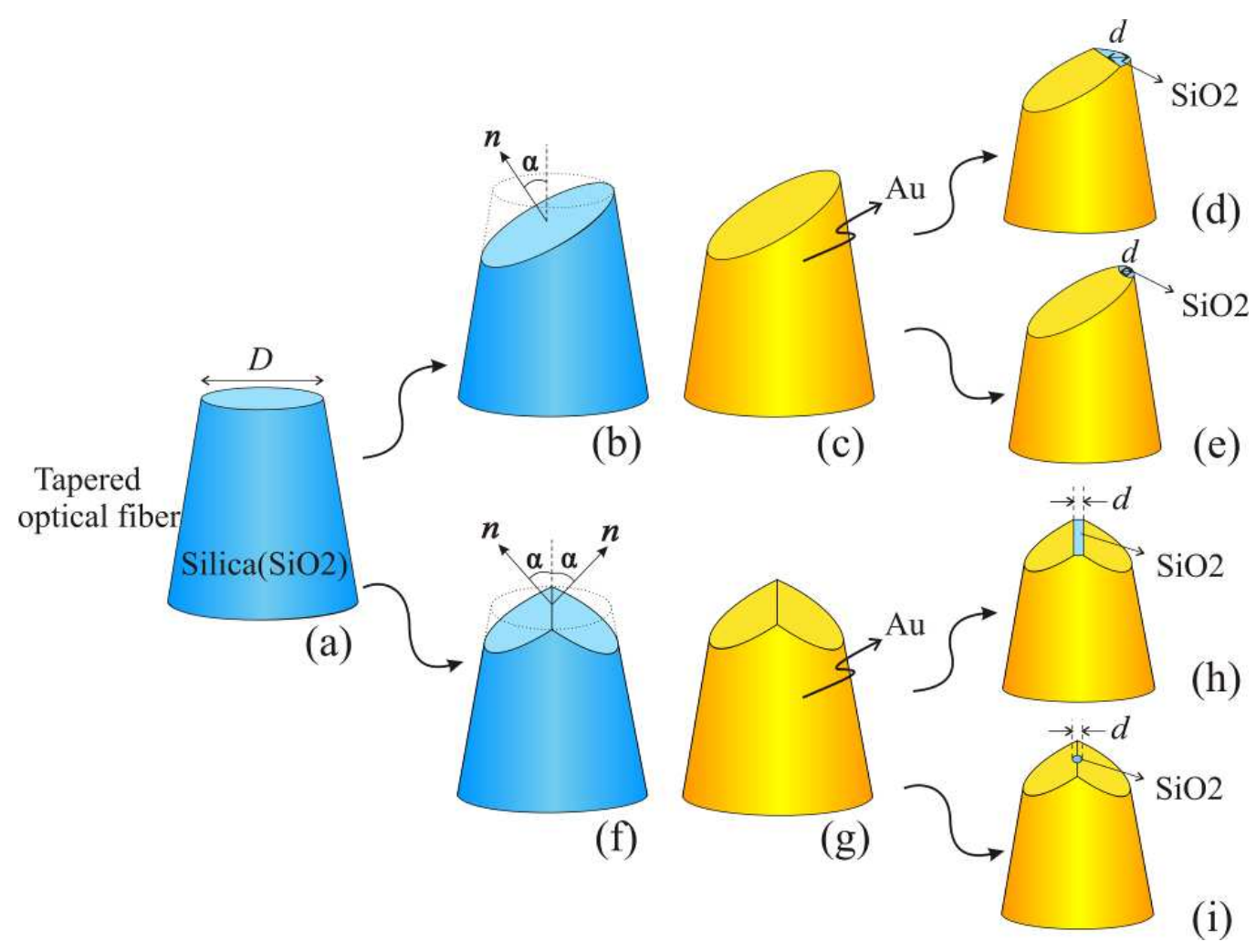

Fig. 14. Schematic of the nanostructured microfiber tip fabrication process. (a) Optical microfiber tips are made using a P-2000 micropipette puller, $D$ is the tip diameter. (b, f) The tips are cut at a slope angle $\alpha$ using focused ion beam (FIB) milling; $n$ is the normal direction to the cleaved surface. (c, g) A thin layer of gold is deposited on the fabricated surface and $(\mathrm{d}, \mathrm{e}, \mathrm{h}, \mathrm{i})$ a small aperture with size $d$ was opened at the apex.

Adiabatic microfiber tips were then nanostructured using a FIB milling system (Fig. 14 (b) and (f)). As FIB is a direct writing technique with resolution less than $10 \mathrm{~nm}$, it offers extreme flexibility and allows for high precision in the nanostructuring. Fig. 15 shows scanning electron microscope (SEM) images of two first fabricated tips: (a) a single-ramp microfiber tip and (b) a wedge microfiber tip. 


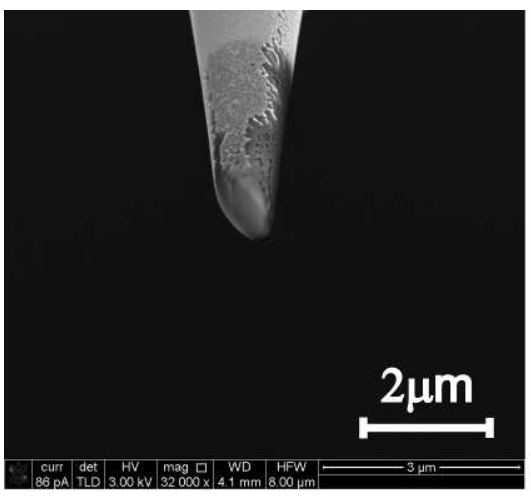

(a)

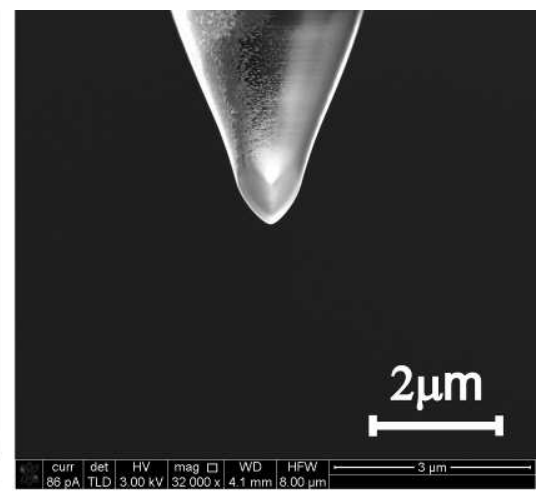

(b)

Fig. 15 SEM images of first milled tips. (a) single-ramp microfiber tip and (b) wedge microfiber tip.

A thin layer of gold was then deposited on the tips using a thermal evaporator to confine light and to avoid charging during processing; indeed, charge accumulation is a major issue and seriously degrades FIB imaging and processing capabilities. Finally, extremely small apertures were finally opened at the tips apexes (Fig. 14 (d), (e), (h) and (i)).

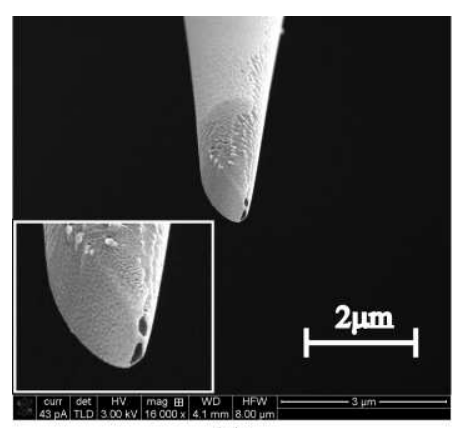

(a)

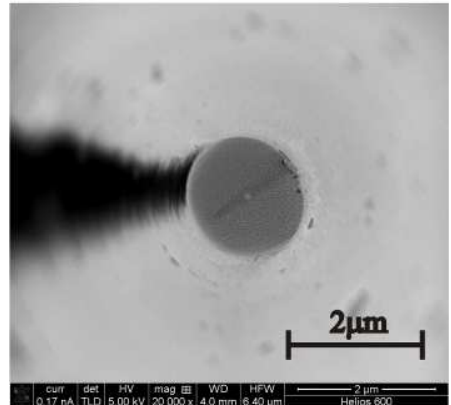

(d)

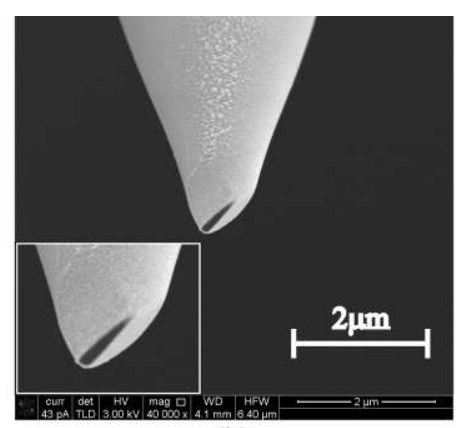

(b)

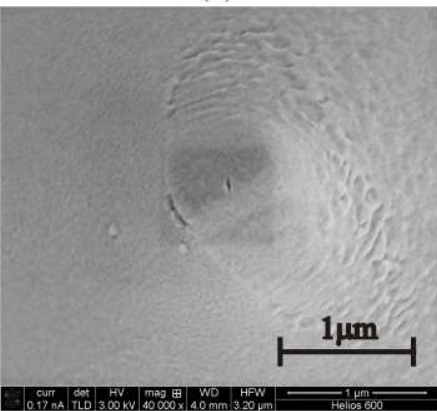

(e)

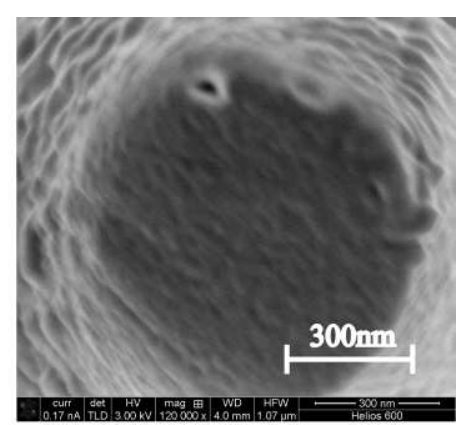

(c)

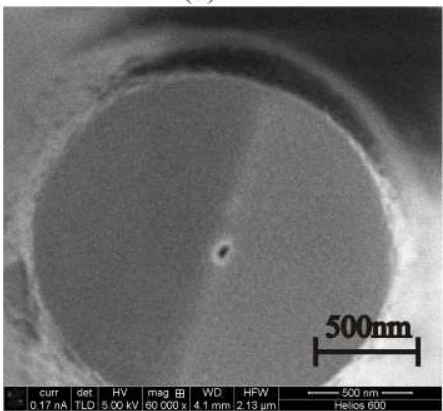

(f)

Fig. 16 SEM images of microfiber tips with nanometer apertures. Tips were cut at slope angle $\alpha$ and apertures (dark regions) were opened at the apexes of gold-coated tips. (a) single-ramp microfiber tip with flat cut aperture size $66.4 \mathrm{~nm}$; (b) wedge microfiber tip with slit aperture size $\sim 84 \mathrm{~nm}$; (c) single-ramp microfiber tip with a hole size $\sim 21 \mathrm{~nm}$; wedge microfiber tips with a hole size (d) $\sim 14 \mathrm{~nm}$, (e) $\sim 13 \mathrm{~nm}$ and (f) $\sim 21 \mathrm{~nm}$. Insets on (a) and (b) are magnification of their apertures. 
Fig. 16 shows the SEM images of final device. Single-ramp tips have apertures made by flat cutting (Fig. 16 (a) with $d \sim 66 \mathrm{~nm}$ ) and hole drilling (Fig. 16 (c) with size $d \sim 21 \mathrm{~nm}$ ), respectively; apertures in the wedge tips are $\sim 84 \mathrm{~nm}$ (Fig. 16 (b)) made by flat cutting and $\sim 14 \mathrm{~nm}$ (Fig. 16 (d)), 13 nm (Fig. 16 (e)) and 21 nm (Fig. 16 (f)) made by hole drilling. Because of the limited FIB stage travel in our fabrication facility, up to now it has been impossible to fabricate the conical microfiber tip as simulated in theoretical analysis.

\section{Characterization}

The devices transmission efficiency was characterized using a supercontinuum (SC) source (FIANIUM Inc., Southampton, UK) delivering $6 \mathrm{~W}$ of $400 f s, 50 \mathrm{~nJ}$ pulses over a very broad range of wavelength from $450 \mathrm{~nm}$ to $1800 \mathrm{~nm}$ to inject light into the samples under test. The SC output fiber pigtail was angle cleaved to avoid back reflection. A modal filter was inserted to minimize the fraction of power in high order modes [66]. Light was then launched into the sample and finally collected using a multimodal pigtail connected to an optical spectrum analyzer (AQ6317, Yokogawa, Japan). XYZ stages were used to align the different fiber components.

Preliminary measurements were carried out without any sample to provide a normalization base for the following spectra and to remove any wavelength dependence related to the source and to the modal filter. Sample spectral measurements were then carried out.

Tips A and B in this characterization are showed in Fig. 16 (a) and (b). They were made by P-2000 pipette puller and cleaved using the FIB system of FEI at slope angle $\alpha=50.4^{\circ}$ and $50^{\circ}$. The cleaved tapers were coated with $<5 \mathrm{~nm}$ chrome and $30 \mathrm{~nm}$ gold and apertures $66.4 \mathrm{~nm}$ and $84 \mathrm{~nm}$ wide were opened by flat cutting in the tip apexes. Chrome was use to ensure a good gold adhesion to the silica tip even at high incident powers [38].

Measurement results for the single-ramp sample A transmission efficiency are summarized in Fig. 17. Three peaks at about $800 \mathrm{~nm}, 1100 \mathrm{~nm}$ and $1500 \mathrm{~nm}$ were observed. The three peaks could come from the presence of different cleaving angles in the tip due to the imprecision in the FIB and/or of multiple modes in the microfiber. The small periodicity noise is probably due to the interference generated at air gaps between the various optical components. The transmission 
efficiency of sample $\mathrm{A}$ is $\sim 10^{-2}$ and several orders of magnitude larger than that observed in common SNOM tips $\left(10^{-4} \sim 10^{-5}\right)$. The spot size of this sample along the smaller axis is as large as $\sim \lambda / 15$. Powers close to $1 \mathrm{~W}$ were injected in the tip without any apparent damage. It is interesting to note that microfiber tips which exploited SPs for focusing could not stand powers of $0.1 \mathrm{~W}$, as the strong field at the metal/air surface probably damaged the microfiber tip surface.

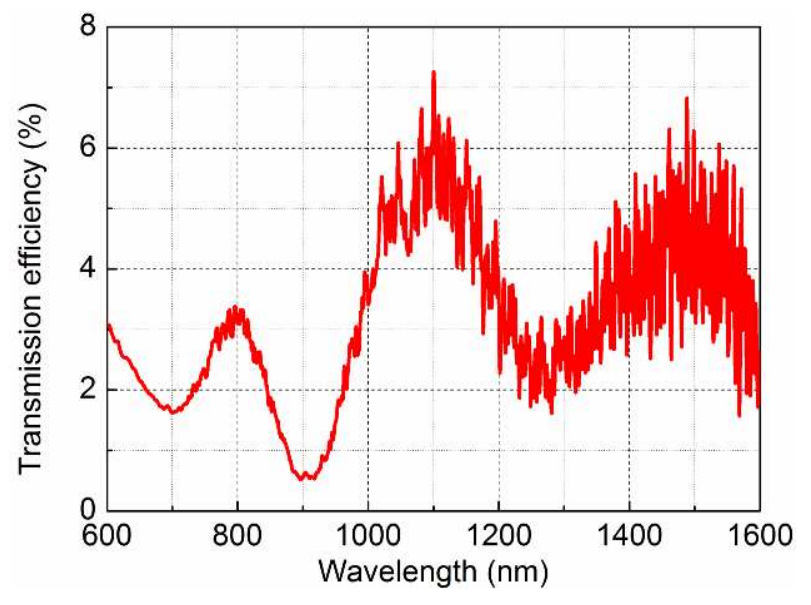

Fig. 17 Transmission efficiency of the single-ramp fiber tip A in Fig. 16 (a).

Fig. 18 shows the normalized transmission of wedge sample $B$, which reaches $\sim 3 \%$ transmission efficiency at $\lambda \sim 750 \mathrm{~nm}$ : the wavelength dependence clearly resembles that predicted by simulations of Fig. 13. As for the results presented in Fig. 17, the small periodicity noise in this graph could be due to the interference generated by air gaps between the various optical components again. The spot size along the smaller axis of this sample is approximately $\lambda / 10$.

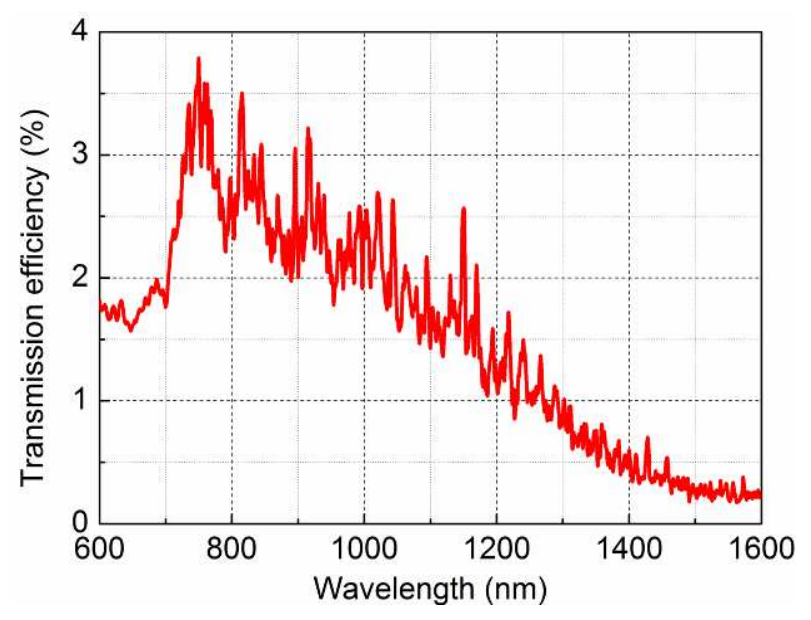

Fig. 18 Transmission efficiency of the wedge fiber tip of Fig. 16 (b). 
The transmission efficiency measured in the experiments is of the same order of magnitude of (indeed a little lower than) those predicted by simulations. The mismatch is probably due to the presence of additional modes in the microfiber tip, to surface/coating nonuniformities, the shape of nanostructured tip difference and to the different polarization used in the simulations and in the experiments. In additional, since we used a multimode fiber to collect the light, the measurement cannot discriminate between the light in the hot spot and "unfocused" light around it.

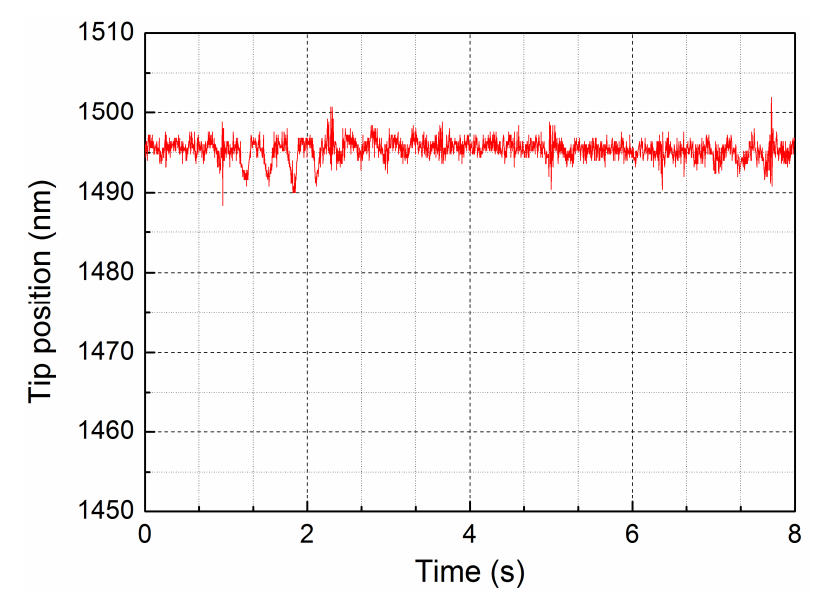

Fig. 19 The tip position as a function of time whilst switching on and off the laser ( $9 \mathrm{~mW}$, switched every $\sim 2 \mathrm{~s})$.

Since the metal-coated microfiber tips may undergo thermal expansion due to the energy absorption by the metal when high laser powers are injected into them, their transmission efficiency (and reliability) can be affected. The study of the tips thermal expansion was carried out using a homemade SNOM using a method reported previously [36] in University College London (London, UK) [67]. A He-Cd laser with $\lambda=325 \mathrm{~nm}$ and power of $9 \mathrm{~mW}$ was launched into the microfiber tip $\mathrm{C}$ shown in Fig. 16 (f) made from a pure silica fiber. The tip, which had been mounted on a tuning fork, was held in close contact with the surface of a bare glass surface using shear force feedback. If, due to absorption, the tip heats up, its length varies by an amount $\Delta h_{t i p}$, and the feedback responds by adjusting the sample position by an equivalent amount. Thus the sample position was followed as a function of the time and used as a measure of $\Delta h_{\text {tip }}$ whilst using a shutter to switch the laser power on and off with $\sim 2 \mathrm{~s}$ period (Fig. 19). From the data, no discernible variation in $\Delta h_{t i p}$ related to the laser power could be detected within the accuracy of 
the system $(\sim 2 \mathrm{~nm})$. This result is remarkable when comparing to measurements made on other fiber probes where $\Delta h_{t i p}$ of up to hundreds of nanometers have been measured for similar injected laser powers, but confirms the good mechanical stability of our tips.

\section{Conclusion}

This paper presented preliminary studies on the efficient sub-wavelength light confinement of high powers in apertured optical microfiber tips. Theoretical simulations were carried out to predict optima, which could provide a reference for the device fabrication suitable for data recording (like AOMR), photolithography and imaging. Devices were manufactured using a P2000 micropipette-puller, an evaporator, and a FIB system. Device transmission spectra were measured with a supercontinuum source and transmission efficiencies of the order of few percent were recorded. The microfiber tips could survive powers as large as $1 \mathrm{~W}$ and thermal expansion measurements showed no shape changes could be detected within the accuracy of the system $(\sim 2$ $\mathrm{nm}$ ) for $9 \mathrm{~mW}$ injected powers. Future optimizations will require the use of conical tips and nearfield measurement to characterize the spot size. Effective confinements to $10 \mathrm{~nm}$ or smaller can be envisaged by decreasing the aperture size and slope angle. Application of this small spot size source can include SNOM, optical recording, photolithography and bio-sensing.

GB gratefully acknowledges the Royal Society (London, U.K.) for his University Research Fellowship.

\section{Reference}

[1] L. Tong, R. R. Gattass, J. B. Ashcom, S. He, J. Lou, M. Shen, I. Maxwell, E. Maxur, Nature 426 (2003) 816-819.

[2] G. Brambilla, J. Opt. 12 (2010) 043001.

[3] G. Brambilla, Opt. Fiber Technol. 16 (2010) 331-342.

[4] M. Born, E. Wolf, Principle of Optics, Springer, New York, 1999.

[5] N. Fang, H. Lee, C. Sun, X. Zhang, Science 308 (2005) 534-537.

[6] A. Salandrino, N. Engheta, Phys. Rev. 74 (2006) 075103. 
[7] V. M. Shalaev, Nat. Photonics 1 (2007) 41-48.

[8] Z. Liu, H. Lee, Y. Xiong, C. Sun, X. Zhang, Science 315 (2007) 1686.

[9] X. Zhang, Z. Liu, Nat. Mater. 7 (2008) 435-441.

[10] Q. Xu, V. R. Almeida, R. R. Panepucci, M. Lipson, Opt. Lett. 29 (2004) 1626-1628.

[11] G. S. Wiederhecker, C. M. B. Cordeiro, F. Couny, F. Benabid, S. A. Maier, J. C. Knight, C.

H. B. Cruz, H. L. Fragnit, Nat. Photonics 1 (2007) 115-118.

[12] X. Wang, J. Fu, X. Liu, L.-M. Tong, JOSA A 26 (2009) 1827-1833.

[13] J. Fu, H. Dong, W. Fang, Appl. Phys. Lett. 97 (2010) 041114.

[14] E. Betzig, J. K. Trautman, Science 257 (1992) 189-195.

[15] H. Heinzelmann, D. W. Pohl, Appl. Phys. A 59 (1994) 89-101.

[16] L. Novotny, D. W. Pohl, B. Hecht, Ultramicroscopy 61 (1995) 1-9.

[17] S. A. Maier, P. G. Kik, H. A. Atwater, S. Meltzer, E. Harel, B. E. Koel, A. A. G. Requicha, Nat. Mater. 2 (2003) 228-232.

[18] W. L. Barnes, A. Dereux, T. W. Ebbesen, Nature 424 (2003) 824-830.

[19] R. Zia, M. D. Selker, P. B. Catrysse, M. L. Brongersma, J. Opt. Soc. Am. A 21 (2004) 24422446.

[20] F. Festy, A. Demming, D. Richards, Ultramicroscopy 100 (2004) 437-441.

[21] V. G. Veselago, Sov. Phys. Uspekhi-USSR 10 (1968) 509-514.

[22] J. B. Pendry, Phys. Rev. Lett. 85 (2000) 3966-3669.

[23] D. R. Smith, W. J. Padilla, D. C. Vier, S. C. Nemat-Nasser, S. Schultz, Phys. Rev. Lett. 84 (2000) 4184-4187.

[24] R. A. Shelby, D. R. Smith, S. Schultz, Science 292 (2001) 77-79.

[25] E. H. Synge, Philos. Mag. 6 (1928) 356-362.

[26] B. Hecht, B. Sick, U. P. Wild, V. Deckert, R. Zenobi, O. J. F. Martin, D. W. Pohl, J. Chem. Phys. 112 (2000) 7761-7774.

[27] D. Courjon, C. Bainier, Rep. Prog. Phys. 57 (1994) 989-1028.

[28] D. Zeisel, S. Nettesheim, B. Dutoit, R. Zenobi, Appl. Phys. Lett. 68 (1996) 2491-2492.

[29] R. Stevenson, R. Riehn, R.G. Milner, D. Richards, E. Moons, D.-J. Kang, M. Blamire, J. Morgado, F. Cacialli, Appl. Phys. Lett. 79 (2001) 833-835.

[30] D. Credgington, O. Fenwick, A. Charas, J. Morgado, K. Suhling, F. Cacialli, Adv. Funct. Mat. 20 (2010) 2842-2847.

[31] W. Ding, S. R. Andrews, S. A. Maier, Phys. Rev. A 75 (2007) 063822.

[32] T. J. Antosiewicz, P. Wrobel, T. Szoplik, 11th International Conference on Transparent Optical Networks Tu.A4.5 (2009) 1-4.

[33] L. Novotny, D. W. Pohl, B. Hecht, Opt. Letts. 20 (1995) 970-972. 
[34] J. A. Veerman, A. M. Otter, L. Kuipers, N. F. van Hulst, Appl. Phys. Lett. 72 (1998) 31153117.

[35] L. Novotny, D. W. Pohl, P. Regli, J. Opt. Soc. Am. A-Opt. Image Sci. Vis. 11 (1994) 17681779 .

[36] R. Riehn, A. Charas, J. Morgodo, F. Cacialli, Appl. Phys. Lett. 82 (2003) 526-528.

[37] F. Renna, D. Cox, G. Brambilla, Opt. Express 17 (2009) 7658-7663.

[38] F. Renna, G. Brambilla, D. C. Cox, IEEE Photonics Technol. Lett. 21 (2009) 1508-1510.

[39] D. A. Parthenopoulos, P. M. Rentzepis, Science 245 (1989) $843 \sim 845$.

[40] E. N. Glezer, M. Milosavljevic, L. Huang, R. J. Finlay, T.-H. Her, J. P. Callan, E. Mazur, Opt. Lett. 21(1996) 2023-2025.

[41] J. F. Heanue, M. C. Bashaw, L. Hesselink, Science 265 (1994) 749-752

[42] D. Psaltis, G. W. Burr, Computer 31 (1998) 52-60.

[43] L. P. Pitaevskii, Sov. Phys. JETP 12 (1961) 1008-1031.

[44] J. P. van der Ziel, P. S. Pershan, L. D. Malmstrom, Phys. Rev. Lett. 15 (1965) 190-193.

[45] A. V. Kimel, A. Kirilyuk, P. A. Usachev, R. V. Pisarev, A. M. Balbashov, Th. Rasing, Nature 435 (2005) 655-657.

[46] C. D. Stanciu, F. Hansteen, A. V. Kimel, A. Tsukamoto, A. Itoh, A. Kirilyuk, Th. Rasing, Phys. Rev. Lett. 98 (2007) 207401.

[47] F. Hansteen, A. Kimel, A. Kirilyuk, T. Rasing, Phys. Rev. Lett. 95 (2005) 047402.

[48] A. V. Kimel, A. Kirilyuk, F. Hansteen, R. V. Pisarev, T. Rasing, J. Phys.: Condens. Matter 19 (2007) 043201.

[49] L. Aigouy, Y. de Wilde, C. Fretigny, Les nouvelles microscopies: A la decouverte du nanomonde, Belin, 2006, pp. 132-234.

[50] P. Moar, F. Ladouceur, L. Cahill, Appl. Opt. 39 (2000) 1966-1973.

[51] P. Hoffmann, B. Dutoit, R.-P. Salathe, Ultramicroscopy 61 (1995) 165-170.

[52] C. Lehrer, L. Frey, S. Petersen, Th. Sulzbach, O. Ohlsson, Th. Dziomba, H. U. Danzebrink, H. Ryssel, Microelectron. Eng. 57-58 (2001) 721-728.

[53] S. Nagai, K. Takehisa, T. Enami, T. Nishisaka, J. Fujimoto, O. Wakabayashi, H. MIzoguchi, A. Takahashi, Appl. Phys. 38 (1999) 7013-7016.

[54] J. E. Bjorkholm, Intel Tech. J. Q3'98 (1998) 1-8.

[55] P. A. Zimmerman, C. van Peski, B. Rice, J. Byers, N. J. Turro, X. Lei, J. Lopez Gejo, V. Liberman, S. Palmacci, M. Rothchild, A. Whitker, I. Blakey, L. Chen, B. Dargaville, H. Liu, J. Photopolym. Sci. Technol. 20 (2007) 643-650.

[56] D. Credgington, O. Fenwick, A. Charas, J. Morgado, K. Suhling, F. Cacialli., Adv. Funct. Mat. 20 (2010) 2842-2847.

[57] Y. Lin, M. H. Hong, W. J. Wang, Y. Z. Law, T. C. Chong, Appl. Phy. A 80 (2005) 461-465 
[58] B. Wu, Science China 54 (2011) 959-979.

[59] R. S. Ghaida, P. Zarkesh-Ha, J. Electron. Test. 25 (2008) 67-77.

[60] G. Vienne, K. Eason, Z. Xie, Y. T. Lim, J. Li, Jpn. J. Appl. Phys. 50 (2011) 09MD03.

[61] A. V. Kimel, A. Kirilyuk, T. Rasing, Laser \& Photon. Rev. 1(2007) 275-287.

[62] N. A. Issa, R. Guckenberger, Plasmonics 2 (2007) 31-37.

[63] J. M. McMahon, S. K. Gray, G.C. Schatz, Nano. Lett. 10 (2010) 3473-3481.

[64] F. J. Garcia de Abajo, J. Phys. Chem. C 112 (2008) 17983.

[65] J. D. Love, W. M. Henry, W. J. Stewart, R. J. Black, S. Lacroix, F. Gonthier, IEE Proc. J 138 (1991) 343-354.

[66] Y. M. Jung, G. Brambilla, D. J. Richardson, Opt. Express 16 (2008) 14661-14667.

[67] A. Ambrosio, O. Fenwick, F. Cacialli, R. Micheletto, Y. Kawakami, P. G. Gucciardi, D. J.

Kang, M. Allegrini, J. Appl. Phys. 99 (2006) 084303 